\title{
Stacking HMW-GS transgenes in bread wheat: Combining subunit 1Dy10 gives improved mixing properties and dough functionality
}

\author{
Elena León ${ }^{a}$, Racha Aouni ${ }^{\mathrm{b}}$, Fernando Piston ${ }^{\mathrm{a}}$, Marta Rodríguez-Quijano ${ }^{c}$, Peter R. Shewry ${ }^{\mathrm{c}}$, \\ Antonio Martín ${ }^{\mathbf{a}}$, Francisco Barro ${ }^{\mathbf{a}, *}$ \\ anstituto de Agricultura Sostenible, CSIC, E-14080-Córdoba, Spain \\ 'Agrasys, S.L, E-08028 Barceiona, Spain \\ " Universidad Politécnica de Madrid, E-28040-Madrid, Spain \\ d Rotiamsted Research, Haipenden, Hertfordsivire AL5 2JQ UK
}

Keywords:

Cereal breeding

1 Ax1

$1 \mathrm{Dx} 5$

1 Dy 10

Anza

Breadmaking qualicy

CM wheat

\begin{abstract}
A B S T R A C T
We have determined the technological properties of four lines containing combinations of three HMWGS transgenes, encoding HMW-GS 1Ax1, 1DX5 and 1Dy10. These lines were produced by conventional crossing of three single transgenic lines of the bread wheat cultivar Anza that contains the endogenous HMW-GS pairs 1Dx2 + 1Dy12 and $1 \mathrm{~B} \times 7^{*}+1 \mathrm{By} 8$ and is null for the Glii-A1 locus. Consequently, the total number of HMW-GS ranged from 4 in the control line Anza to 7 in line T618 which contains all three HMW-GS transgenes. The lines were studied over two years using a range of widely used grain and dough testing methods. All lines with transgenic subunits showed higher levels of glutenin proteins than the Anza control, and these differences were highly significant for lines T616, T617 and T618, containing. respectively, the transgenes encoding HMW-GS $1 \mathrm{Ax} 1$ and $1 \mathrm{Dy} 10,1 \mathrm{D} \times 5$ and $1 \mathrm{Dy} 10$ and $1 \mathrm{~A} \times 1.1 \mathrm{Dx} 5$ and 1Dy10. These increases in glutenin levels are compensated by lower levels of gliadins present in transgenic lines. These changes affected the ratio of polymeric to monomeric gluten proteins (poly:mono), the ratio of HMW-GS to LMW-CS (HMW:LMW) and the contents of individual 1Ax, 1Bx, 1By, 1Dx and 1Dy subunits. Transgenic lines expressing subunit 1Dy 10 together with $x$-type subunits (T616, T617 and T618) were superior to line $T 606$, which had only increases in $x$-type subunits. In particular, the combination of transgenic subunits 1Dx5 and 1Dy 10 (line T617) gave better dough rheological properties than the other combinations of transgenic subunits. For example, dough development time and stability were increased by 3.5-fold and 8.5 -fold, respectively, while the mixing tolerance index (MTI) was decreased by 3.3-fold in line T617 with respect to the control line. Alveograph analyses showed that all four transgenic combinations had increased $P$ values compared to the Anza control but subunit $1 \mathrm{D} \times 5$ greatly reduced the extensibility $(L)$. These results show that stacking HMW-GS transgenes by conventional crossing is a valid strategy for the improvement of wheat quality, with different effects being related to the different HMWGS combinations.
\end{abstract}

\section{Introduction}

The unique processing properties of wheat result from the unusual biomechanical properties of the gluten proteins, which form a network conferring elasticity and extensibility to the dough. These viscoelastic properties allow wheat to be used for many food products such as bread, cake, biscuits, pasta and noodles. Gluten proteins consist of monomeric gliadins and polymeric glutenins. Gliadins are single chain molecules which form only intra-chain disulphide bonds. In contrast, the glutenin subunits form both inter- and intra-chain disulphide bonds. The high molecular weight glutenin subunits (HMW-GS) of wheat play an important role in determining the functional properties of wheat dough. Bread wheat contains six HMW-GS genes, with tightly linked pairs of genes encoding $\mathrm{x}$-and $\mathrm{y}$-type subunits being present at each of the Glu-A1, Glu-B1, and Glu-D1 loci on the long arms of chromosomes 1A, 1B, and 1D, respectively. Allelic differences in the HMW-GS composition result in effects on the structures and properties of the glutenin polymers and hence on breadmaking quality (Payne, 1987; Shewry et al., 2003b). In particular, HMW-GS $1 \mathrm{Ax} 1$ and $1 \mathrm{Dx} 5+1 \mathrm{Dy} 10$, encoded by chromosomes $1 \mathrm{~A}$ and $1 \mathrm{D}$, respectively, are associated with strong dough and good breadmaking quality. 
It is therefore not surprising that genes for HMW-GS have been identified as targets for expression in transgenic wheat (Altpeter et al., 1996; Barro et al., 1997; Blechl and Anderson, 1996) to develop cultivars with new HMW-GS combinations and hence with improved or novel functional properties. Differential effects of transgenes encoding HMW-GS 1Ax1, 1Dx5 and 1Dy10 on gluten properties have been reported (Barro et al., 2003; Blechl et al., 2007; Darlington et al., 2003). Furthermore, the introgression of transgenes into a commercial cultivar confirmed differential effects of subunits $1 \mathrm{Ax} 1$ and $1 \mathrm{Dx} 5$ (Field et al., 2008). Thus, expression of subunit $1 \mathrm{Ax} 1$ in transgenic wheat resulted in lines with improved rheological properties while lines expressing subunit $1 \mathrm{D} \times 5$ resulted in unsuitable properties for breadmaking. In particular, high levels of expression of subunit $1 \mathrm{Dx} 5$ resulted in greatly increased dough strength, being too strong for conventional breadmaking (Alvarez et al., 2001; Darlington et al., 2003; Rooke et al., 1999). However, in these studies the HMW-GS 1Dx5 transgene was expressed in the absence of the HMW-GS 1Dy10 transgene. HMW-GS 1Dx5 and 1 Dy 10 are always expressed as an allelic pair and the extreme effects of the $1 \mathrm{Dx} 5$ transgene alone on dough elasticity could have resulted, at least in part, by disturbance of the usual balance of $x$-type and y-type HMW-GS (Butow et al., 2003).

More recently, we reported the transformation of a commercial wheat cultivar Anza with HMW-GS genes to add subunits $1 \mathrm{Ax} 1$, $1 \mathrm{Dx} 5$ or $1 \mathrm{Dy} 10$ to a genetic background that contains endogenous genes for subunits $1 \mathrm{Dx} 2$ and $1 \mathrm{Dy} 12$ instead of the allelic genes for subunits 1Dx5 and 1Dy10 (León et al., 2009). The expression of all three individual subunits resulted in increased dough strength. However, the expression of subunit 1Dy10 had a greater effect on potential breadmaking quality than that of subunits $1 \mathrm{Ax} 1$ and 1Dx5. We have now used conventional crossing of these lines to develop cultivars combining two and three HMW-GS transgenes in the same genetic background. This allows the effects of the interactions between the pairs of subunits to be determined in order to identify optimum combinations of HMW-GS for future studies.

\section{Material and methods}

\subsection{Plant material}

Four transgenic lines and two control lines were used in this work. Transgenic lines were obtained by crossing lines T580, T581 and T590 (Fig. 1) containing the transgenes for the HMW-GS 1Dx5, $1 \mathrm{Ax} 1$ and 1Dy10, respectively (León et al., 2009). Control lines were the bread wheat crs Anza and Perico. Anza is the parent of the transgenic lines and Perico is a high quality commercial variety included for comparison as it contains HMW-GS $1 \mathrm{Ax} 1,1 \mathrm{Dx} 5$ and 1Dy10. Homozygous progeny of plants containing HMW-GS transgenes were identified by SDS-PAGE of endosperm proteins by single half-seed descent (see below). Homozygous lines were selfpollinated and analysed over two years, using a randomized complete block design with two replicates, as described by Barro et al. (2002).

\section{Protein and SDS-PAGE analysis}

The protein content of flour was calculated from the Kjeldahl nitrogen content ( $\% \mathrm{~N} \times 5.7)$ and expressed on a dry matter basis. Seeds were crushed into a fine powder and used to extract the endosperm storage proteins. Gliadins were extracted in $60 \%(\mathrm{v} / \mathrm{v})$ aqueous ethanol using a rotary shaker for $\mathbf{4 0} \mathrm{min}$. Samples were centrifuged at $13,000 \mathrm{~g}$ for 5 min and the supernatant collected. Glutenins were extracted as described by Shewry et al. (1995). For densitometry, glutenins from 30 individual seeds per line and year (60 seeds in total) were separated by SDS-PAGE gels and analysed using a Kodak Image Station 440CF and Kodak 1D Image Analysis Software (Kodak Limited, Boundary Way, Hemel Hempstead, Herts HP2 7YU, UK) using the SDS-PAGE Molecular Weight Standards from Bio-Rad (Bio-Rad Laboratories, 28109 Alcobendas, Madrid) as reference.

\subsection{SE-HPLC}

Size-exclusion high petformance liquid chromatography (SEHPLC) was carried out on three biological samples per line and year with two replicates each ( 12 measurements) at the proteomics service of the University of Cordoba (Cordoba, Spain) as described by Morel et al. (2000) and Larroque et al. (2000) with some modifications. Flour (120 mg) was suspended in $15 \mathrm{ml} 0.5 \%(\mathrm{v} / \mathrm{v})$ SDS-phosphate buffer (pH 6.9) and dispersed at $60^{\circ} \mathrm{C}$ for $80 \mathrm{~min}$ using a rotary shaker. The dispersed flour samples were sonicated (90 s at 70\% power setting) to extract total proteins. After centrifugation, the supernatant was filtered through a $0.45 \mu \mathrm{m}$ PVDF filter. Analyses were performed on a BioBasic C4 column (Thermo Fisher Scientific S.LU, 28108 Alcobendas, Madrid). Proteins were detected by absorption at $214 \mathrm{~nm}$.

\subsection{Grain traits}

Thousand seed weight was determined on two samples of 1000 seeds from each plot per line and year with two replicates each (eight measurements). Test weight expressed in grams per liter was determined by weighing $100 \mathrm{ml}$ of cleaned grains with four replicates per line and year (eight measurements).

\subsection{Quality traits}

The SDS sedimentation test was carried out on two different samples per line and year with two replicates each (eight measurements) using $1 \mathrm{~g}$ of wholemeal per line and year as described by Dick and Quick (1983).

For Alveograph analysis, approximately $0.8 \mathrm{~kg}$ samples of seeds per line and year were conditioned to $16 \%$ moisture content and milled with a Chopin CD1 mill (Chopin technologies, 92396 Villeneuve-la-Garenne Cedex, France). Alveograph tests were performed and the dough properties determined in two samples per year (four measurements) according to standard ICC method no. 121 (ICC, 1992a).

For Farinograph analysis, approximately $0.2 \mathrm{~kg}$ samples of seeds per line and year were milled with a Perten 3100 mill (Perten Instruments, SE-141 05 Kungens Kurva, Sweden) and mixed using a $50 \mathrm{~g}$ mixer for standard Farinograph analysis (Brabender GmbH \& Co. KG, Germany) test. The Farinograph characteristics were determined in two different samples per year with two replicates (eight measurements) according to standard ICC method no. 115/1 (ICC, 1992b).

Dough mixing properties were determined with a $10 \mathrm{~g}$ Mixograph (National Manufacturing Co., Lincoln NE) as described by Finney and Shogren (1972). Samples were mixed to optimum water absorption following 54-40A method (AACC, 2000). The Mixograph characteristics were determined on two samples per year (four measurements).

\subsection{Statistics}

Data were analysed using the SPSS version 11.0 statistical software package (SPSS Inc., Chicago, Illinois, USA). The general analysis of variance and the least significant difference pairwise comparisons of means were used to determine significant differences. 

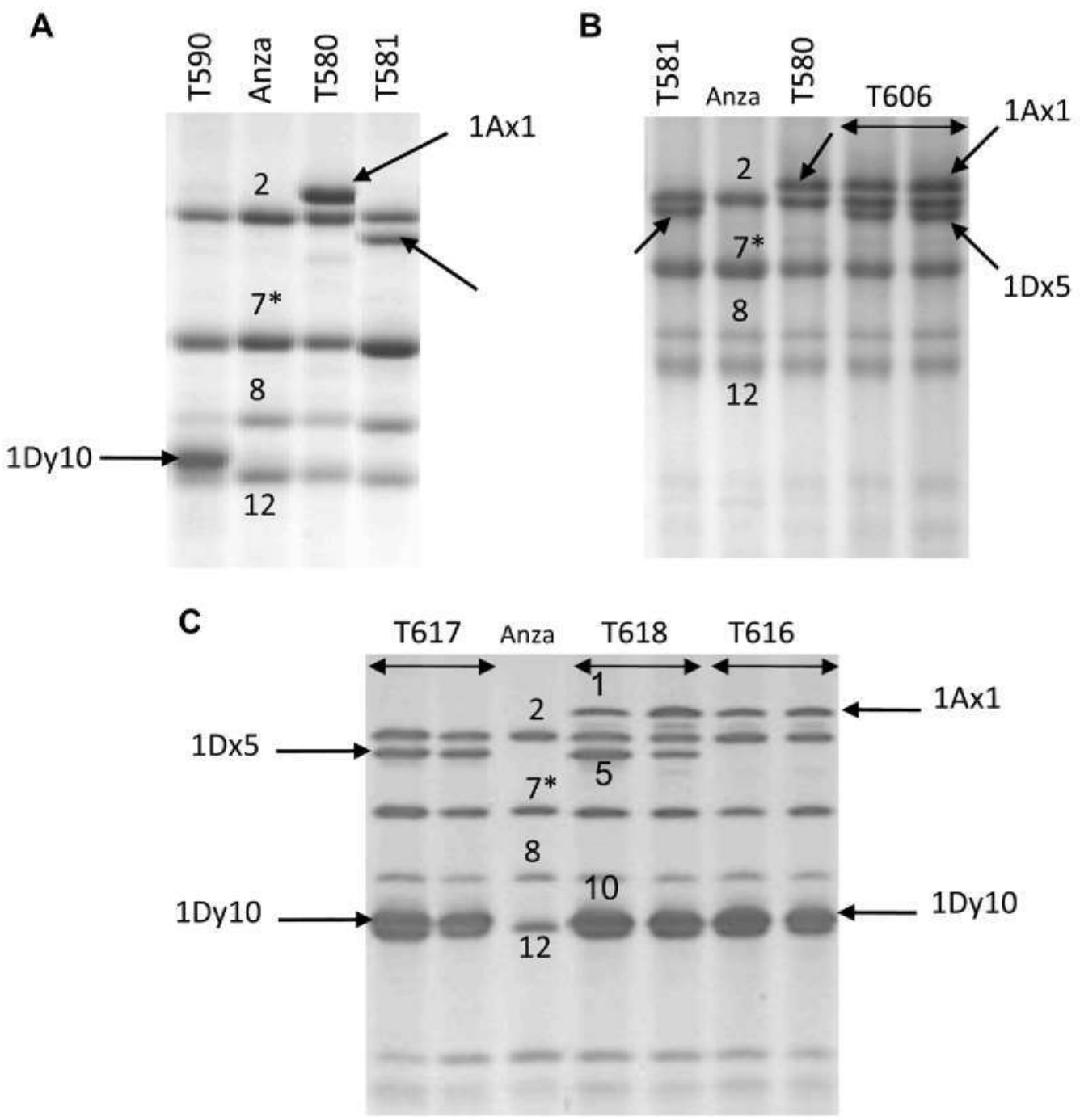

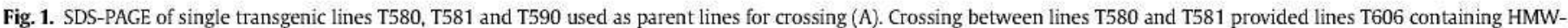

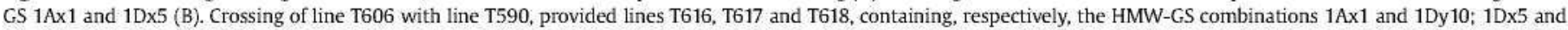
1Dy10; and 1Ax1, 1Dx5 and 1Dy10 (C). The location of the HMW-GS native to Anza (1Dx2, 1Bx7*, 1By9 and 1Dy12) are indicated. Transgenic HMW-GS is arrowhead.

\section{Results and discussion}

The HMW-GS of wheat are major determinants of breadmaking quality, in particular, the subunit pair 1Dx5+1Dy10 and subunit $1 \mathrm{Ax} 1$ have high quality scores (Payne, 1987; Shewry et al., 2003b). We reported the effects of the HMW-GS $1 \mathrm{Ax} 1,1 \mathrm{Dx} 5$ and $1 \mathrm{Dy} 10$ on the breadmaking quality of the bread wheat cultivar Anza that contain the HMW-GS pairs $1 \mathrm{Dx} 2+1 \mathrm{Dy} 12$ and $1 \mathrm{Bx} 7^{*}+1 \mathrm{By} 8$ and is null at the Glu-A1 locus (León et al., 2009). These lines were used as parents (Fig. 1A) for conventional crossing to obtain four hybrid lines, three expressing pairs of transgenic subunits and one expressing all three transgenic subunits. This is therefore the first report on the characterization of wheat expressing combinations of these three transgenic subunits in the same genetic background. Thus, crossing of lines T580 and T581 gave line T606 containing subunits $1 \mathrm{Ax} 1$ and $1 \mathrm{Dx} 5$ (Fig. 1B) and further crossing of line T606 with line T590 gave lines T616, T617 and T618 containing subunits $1 \mathrm{Ax} 1$ and $1 \mathrm{Dy} 10 ; 1 \mathrm{Dx} 5$ and $1 \mathrm{Dy} 10$; and $1 \mathrm{Ax} 1,1 \mathrm{Dx} 5$ and $1 \mathrm{Dy} 10$, respectively (Fig. 1C). Single seed descent was used to obtain homozygotes by screening half-seeds on SDS-PAGE gels. Lines were multiplied by self-pollination and analysed for two years as described by Barro et al. (2002). Technological properties and the potential breadmaking quality of the flours were determined for each of two years of field growths using a range of widely used grain and dough testing methods.

\subsection{Characteristics of the storage proteins}

Table 1 compares the characteristics of the transgenic lines with the control line Anza, which contains four endogenous HMW-GS: $1 \mathrm{Bx} 7^{*}+1 \mathrm{By} 8$ and $1 \mathrm{Dx} 2+1 \mathrm{Dy} 12$. In addition, a commercial variety of bread wheat of good breadmaking quality, Perico expressing five HMW-GS (1Ax1, 1Bx17+1By18,1Dx5+1Dy10), was included as reference line. Consequently, the total number of HMW-GS ranged from 4 for control line Anza to 7 for line T618. Anza and lines T616 and T617 had equal number of $\mathrm{x}$ - and $\mathrm{y}$-type subunits while lines T606 and T618 had higher numbers of $\mathrm{x}$-type subunits. Although crossing of the transgenic lines resulted in hybrid lines containing increasing numbers of HMW-GS (Table 1), this did not have a significant effect on the total flour protein content compared to the Anza control (Table 1). In fact, line T618 expressing 7 subunits had similar total flour protein content to the control line Anza. Although the three lines expressing transgenic subunit pairs (T606, T616 and T617) had higher protein contents than Anza and line 


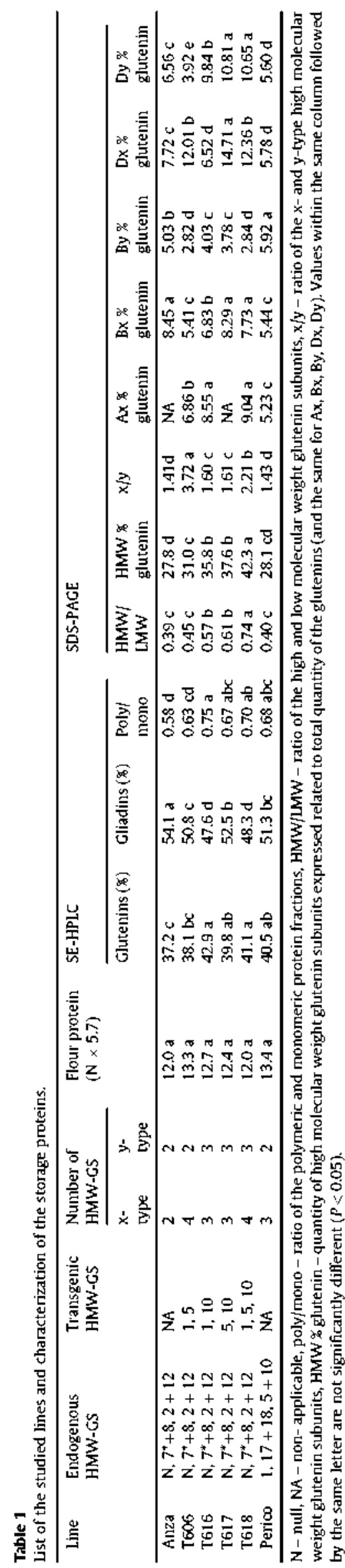

Table 2

Technological properties of the transgenic and control lines.

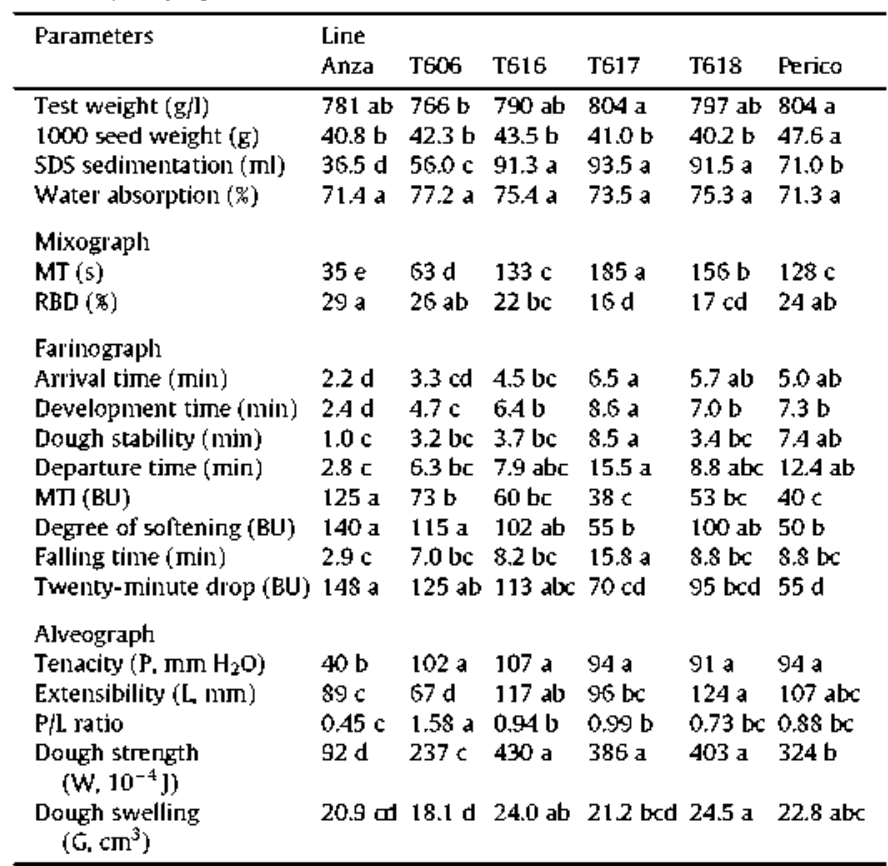

Values within the same parameter followed by the same letter are not significantly different $(P<0.05)$.

T618, these differences were not significant according to the LSD test (Table 1). Rakszegi et al. (2005) also reported no significant increase in the protein content of transgenic wheat expressing an increased amount of HMW-GS.

The proportions of glutenins and gliadins and the ratio of polymeric/monomeric gluten proteins were determined by sizeexclusion HPLC (SE-HPLC). The combinations of transgenic subunits significantly affected the proportions of protein fractions in the endosperm of wheat lines. All transgenic lines had higher proportions of glutenins than the Anza control $(37.3 \%)$, with the highest proportion being in line T616 (42.9\%). These differences were highly significant for lines T616, T617 and T618 (Table 1). In contrast, line T618 which expressed all three transgenic subunits (1Ax1,1Dx5 and 1Dy10) had a similar proportion of glutenins to the commercial variety Perico which expressed endogenous forms of the same three subunits (Table 1). These increases in proportions of glutenins are compensated by the lower proportions of gliadins present in transgenic lines. Thus, the proportion of gliadins varied significantly among lines expressing transgenic subunits, being in all cases lower than in the Anza control (Table 1 ). These changes in the proportions of the two major protein fractions affected the polymer:monomer ratios. The ratio of polymeric and monomeric fractions was higher for lines T616 and T618 with the Anza control showing the lowest value (Table 1). Finally, the proportions of glutenins, and the ratio of polymer:monomer were similar between line T618, expressing transgenic subunits $1 \mathrm{Ax} 1,1 \mathrm{Dx} 5$ and 1Dy10, and the commercial variety Perico that expresses endogenous forms of the same subunits. In contrast, the proportion of gliadins present in line T618 was significantly lower than in Perico (Table 1).

The differences in the proportions of glutenins are reflected in the proportions of total HMW-GS and of individual Ax, Bx, By, Dx and Dy subunits (Table 1). Thus, the HMW-GS:LMW-GS ratio and proportions of HMW-GS in the glutenin fraction were increased in all hybrid lines with respect to the Anza control. These increases were significant for lines T616, T617 and T618 but not for line T606, expressing subunits $1 \mathrm{Ax} 1$ and $1 \mathrm{Dx} 5$ (Table 1 ). 

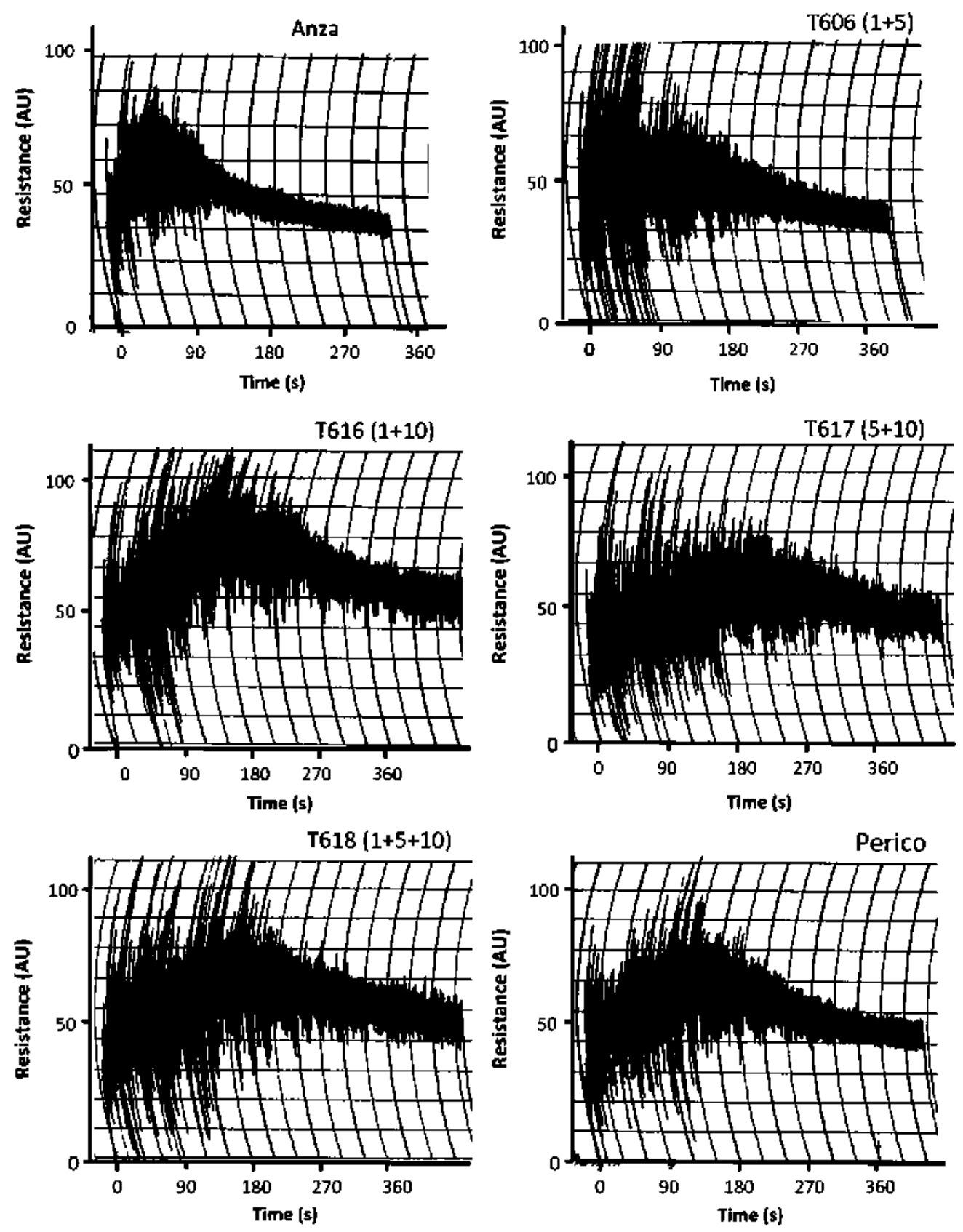

Fig. 2. Mixograph curves of the doughs prepared from non-transformed controls Anza and Perico and those from flours from transgenic lines T606, T616, T617, T618.

In comparison with the Anza control, the expression of subunits $1 \mathrm{Ax} 1$ and $1 \mathrm{Dx} 5$ in line T606 was associated with decreases in the proportions of $1 \mathrm{Bx}, 1 \mathrm{By}$ and $1 \mathrm{Dy}$ subunits and with increases in $1 \mathrm{Ax}$ and $1 \mathrm{Dx}$ subunits. Consequently, the $\mathrm{x}: \mathrm{y}$ ratio was increased significantly in this line (Table 1 ). The expression of subunits $1 \mathrm{Ax} 1$ and 1 Dy 10 in line T616 was also associated with decreases in the proportions of $1 \mathrm{Bx}, 1 \mathrm{By}$ and $1 \mathrm{Dx}$ subunits but with increases in the $1 A x$ and $1 D y$ subunits. Thus, the $x: y$ ratio for line $T 616$ was significantly lower than that of line T606 (Table 1). The expression of subunits $1 \mathrm{D} \times 5$ and $1 \mathrm{Dy} 10$ in line T617 was associated with a decrease in subunit $1 \mathrm{By} 8$ but not in subunit $1 \mathrm{Bx} 7^{*}$ and with increases in 1Dx and 1Dy subunits. Line T617 also expressed one $\mathrm{x}$ and one $y$-type subunit, as T616, and the $x: y$ ratio was similar to that of line T616 and also comparable to that of the Anza control (Table 1). Finally, the expression of two $x$ - and one y-type transgenic subunits in line T618 was associated with a decrease in subunit $1 \mathrm{By} 8$ but not in subunit $1 \mathrm{Bx} 7^{*}$ and with increases in $1 \mathrm{Ax}$, $1 \mathrm{Dx}$ and 1Dy subunits. In this case, the expression of two x-type subunits significantly increased the $x$ :y ratio (Table 1 ).

Considering the data for all four hybrid lines, it is clear that the stacking of HMW-GS transgenes did not increase the total protein content but increased the proportions of the individual HMW-GS encoded by the transgenes. However, differential effects on the proportions of individual endogenous HMW-GS were observed. In most cases the proportions of endogenous subunits were decreased which was probably largely compensatory, reflecting competition for amino acids for protein synthesis. However, co-suppressive effects on individual HMW-GS genes cannot be ruled out. Notably, the proportion of subunit 1By8 was decreased in lines expressing all of the transgenes ( $1 \mathrm{Ax} 1,1 \mathrm{D} \times 5$ and $1 \mathrm{Dy} 10$ ) whereas the 
proportion of subunit $1 \mathrm{Bx} 77^{*}$ was only significantly reduced in two lines (T606 and T616) that both expressed the subunit 1Ax1 transgene. Because the individual HMW-GS differ in their numbers and distributions of cysteine residues, the transgenic lines would be expected to differ in their glutenin polymer structure, with resulting effects on their processing properties.

\section{Breadmaking quality}

Test weight ranged from a maximum of 804 for line T617 to a minimum of 766 for line T606. However, these differences were not significant according to the LSD test. The thousand seed weight was similar for all transgenic lines and the Anza control, with no significant differences among them. The highest values for the SDS sedimentation test were $91.3,93.5$ and 91.5 for lines T616, T617 and T618, respectively, which were significantly different to the values for line T606 and Anza. These increases in SDS sedimentation values imply improved potential breadmaking quality. Although water absorption was higher for all four transgenic lines, the differences were not statistically significant (Table 2 ).

The mixing properties of the dough were determined using a $10 \mathrm{~g}$ Mixograph (Fig. 2) and a Brabender Farinograph (Fig. 3) while the dough viscoelastic properties were determined using a Chopin Alveograph (Fig. 4). The Mixograph is widely used in cereal research as it measures a range of theological parameters that relate to the behaviour of the dough in breadmaking and other food processing systems. The machine measures the resistance as the dough is mixed, the most important parameters being the mixing time (MT, time to maximum resistance) and the resistance break down (RBD, the loss of resistance on over-mixing). In general, strong doughs have long mixing times and low resistance breakdown. The highest value for MT (185s) was for line T617 (expressing the transgenic subunits 1Dx5 and 1Dy10) which was significantly different to the values for the other lines. Line T617 also gave the lowest value for RBD (Table 1). The Mixograph therefore showed that the combination of transgenic subunits $1 D \times 5$ and 1Dy10 gave better mixing properties than the other combinations of transgenic subunits.

The Farinograph characteristics of the transgenic lines are shown in Table 2 and Fig. 3. The parameters provide data on the consistency of the dough (Table 2). All four lines expressing transgenic HMW-GS combinations showed higher values than the Anza control for arrival time, development time, dough stability, departure time and falling time, and lower values for mixing tolerance index, degree of softening and 20-minute drop (Table 2). This indicates that doughs formed from these four lines were stronger than dough from the Anza control. Furthermore, line T617, expressing subunits $1 \mathrm{Dx} 5$ and 1Dy 10 , gave stronger dough than the other three transgenic lines. For example, the dough development time and stability were increased by 3.5 -fold and 8.5 -fold, respectively, in line T617 with respect to the control line. Dough stability is an indicator of the overall quality of the protein in the flour and indicates the time during which the dough maintains the maximum consistency. The mixing tolerance index (MTI) is an indicator of how well the dough will perform during the critical final stages of mixing and is related to strength (Shuey, 1984). Thus, a high MTI means that the dough will tend to break down quickly while a low MTI may indicate that the dough will require longer mixing to fully develop. The MTl was decreased by 3.3 -fold in line $\mathrm{T} 617$ with respect to the control line. The degree of softening varied
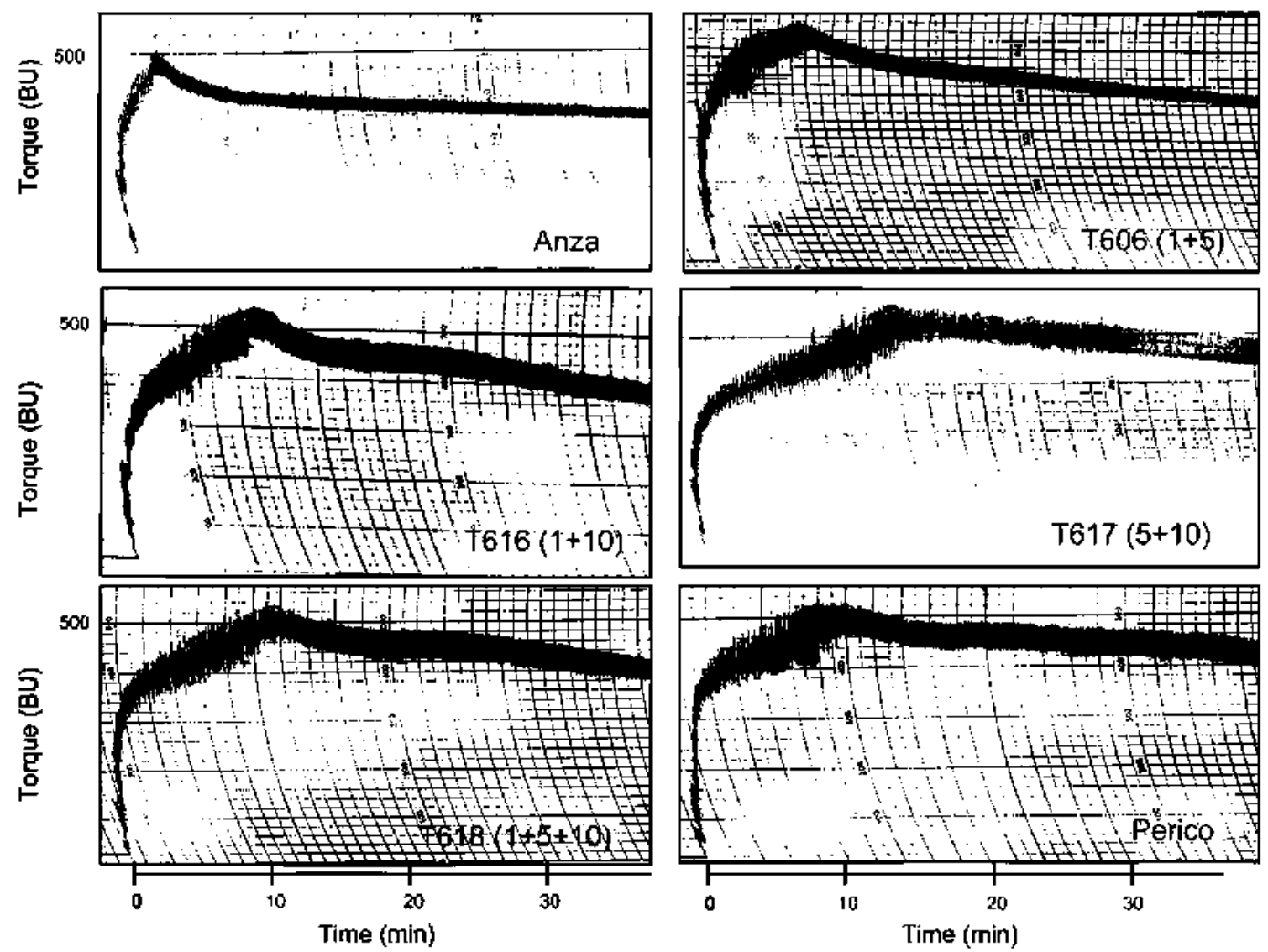

Fig. 3. Farinograms of the doughs prepared from non-transformed controls Anza and Perico and those from flours from transgenic lines T606, T616, T617, T618. 

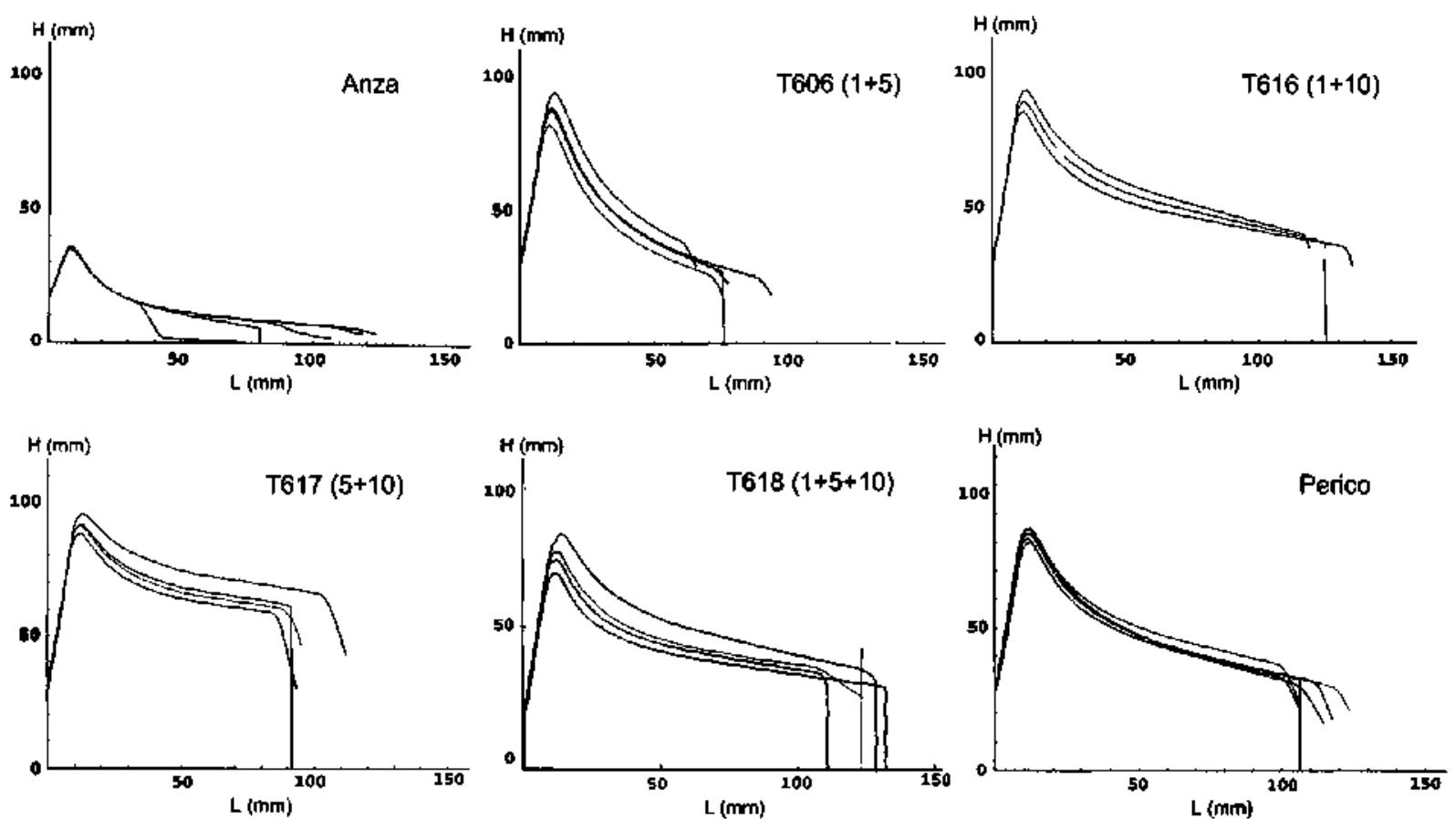

Fig. 4. Alveograph curves of the doughs prepared from non-transformed controls Anza and Perico and those from flours from transgenic lines T606, T616, T617, T618.

from 125 (BU) in the control line to 55 (BU) in the line expressing subunits 1 Dx5 and 1Dy10. Higher values of degree of softening, measured in Brabender units, are characteristic of weak flours and lower values of strong flours. Therefore, the combination of transgenic subunits $1 \mathrm{D} \times 5$ and $1 \mathrm{Dy} 10$ resulted in better Farinográph performance than the other transgenic combinations. It is interesting that the combination of all three subunits in the same background (line T618) did not give better values but values similar to that of line T616 expressing subunits $1 \mathrm{Ax} 1$ and $1 \mathrm{Dy} 10$. This is consistent with the suggestion that a correct balance of $x$-type:ytype subunits is important as well as the absolute amounts.

A comparison of line T618, which expresses all three transgenic subunits, with the commercial variety Perico, which contains the same endogenous $1 \mathrm{~A}$ and $1 \mathrm{D}$ subunits but different $1 \mathrm{~B}$ subunits, is also shown in Table 2 . The values for development time of Perico and T618 were not significantly different. However, dough stability was higher for Perico than that for line T618 while the degree of softening and 20-minute drop were lower for Perico than that for line T618. These differences probably reflect differences in the proportions of subunits $1 \mathrm{Ax} 1,1 \mathrm{Dx} 5$ and $1 \mathrm{Dy} 10$ between the genotypes, with the ratio of $x$-type:y-type subunits being higher in line T618 than in Perico (Table 1).

The Chopin Alveograph simulates the expansion of bubbles in fermenting dough and as such, the parameters derived from this test indicate how the dough will behave during fermentation. The most important parameters obtained from an alveogram are the tenacity $(P)$ of the dough (expressing the resistance of the dough to deformation), the dough extensibility ( $L$ ), the $P / L$ ratio (which gives a general indication of the viscoelastic properties of the dough) and the deformation energy $(W)$, which is proportional to the energy required for deformation. This $W$ value is used to measure the dough strength by bakers. In general, low $W$ values are related to weak flours and high values to strong flours. The $P$ values were similar for the four transgenic lines, but significantly higher with respect to the Anza control (Table 2). As shown in Fig. 4, the combination of the subunits $1 \mathrm{Ax} 1$ and $1 \mathrm{Dx} 5$ in line T606 gave an increase in the $P$ value and a decrease in the length of the curve $(L)$ with respect to the Anza control (Table 2). Nevertheless, line T616, expressing subunits $1 A x 1$ and 1Dy10, did not differ in $P$ value with respect to line T606. The extensibility $(L)$ of line T616 was increased with respect to line T606 (Fig. 4). However, line T617, expressing the combination of transgenic subunits $1 \mathrm{Dx} 5$ and $1 \mathrm{Dy} 10$, showed decreased extensibility $(L)$ in comparison to the Anza control and line T616. On the other hand, the presence of the three transgenic subunits, 1Ax1, 1Dx5 and 1Dy10, in line T618 was associated with slightly increased extensibility (L) with respect to lines T606 and T617 (Fig. 4). The lowest and highest values for the P/L ratio were for Anza and line T606 (subunits 1 and 5), respectively, while it was similar for lines T616 and T617 (Table 2). Deformation energy ( $W$ ) was significantly higher for lines T616, T617 and T618 than for the Anza control and line T606. Finally, comparison of line T618 and Perico showed significant differences in deformation energy $(W)$ which was higher in line $T 618$, while the $P / L$ ratio was similar (Table 2). The Alveograph results showed that all four transgenic combinations had increased $P$ values compared to the Anza control but that subunit $1 \mathrm{D} \times 5$ had a greater effect, reducing extensibility $(L)$. Similar results were reported for subunit 1Dx5 expressed as a single transgene in the same background (León et al., 2009). The addjtional cysteine residue present in the repetitive domain of subunit $1 \mathrm{Dx} 5$ has been suggested to provide an additional site for interchain bonds between HMW-GS (Shewry et al., 2003a). This would increase the cross-linking of the glutenin network with respect to that caused by subunit 1 Dy 10 , reducing extensibility.

\section{General discussion}

In this study, subunits $1 \mathrm{Ax} 1,1 \mathrm{D} \times 5$ and $1 \mathrm{Dy} 10$ were combined in a cultivar of low breadmaking quality which provides an appropriate background to explore their effects on dough properties. This is the first report on the characterization of all three HMW-GS combinations in the same wheat background. All four HMW-GS combinations had significant effects on the composition and functional properties of the gluten fraction. These presumably result from impacts on the structure and properties of the glutenin 
proteins and include effects on the amounts of endogenous subunits and on the ratios of x-type:y-type subunits, HMWGS:LMW-GS, and the ratio of polymeric:monomeric gluten proteins. Studies using the Alveograph, Mixograph and Farinograph indicated that all four transgenic combinations should have better breadmaking quality than the control line. These increases in quality were associated with higher proportions of glutenins and polymeric proteins and lower proportions of gliadins, as previously reported (Blechl et al., 2007; Rakszegi et al.. 2005). Comparisons of the various HMW-GS combinations showed that the combination of subunits $1 \mathrm{Dx} 5$ and 1 Dy 10 resulted in the greatest increase in quality. In contrast, the combination of subunits $1 \mathrm{Ax} 1$ and $1 \mathrm{Dx} 5$ gave the poorest properties. The line with this combination (T606) had the highest ratio of $x$-type:y-type subunits indicating that an appropriate balance of the two subunit types is required (Butow et al., 2003). However, no evidence of "overstrong" properties associated with the expression of the subunit $1 \mathrm{D} \times 5$ transgene, with negative effects on breadmaking quality, was obtained, contrasting with reports of the expression of this transgene in other genotypes (Barro et al., 2003; Darlington et al., 2003; Rooke et al., 1999). However, this may result from the fact that the proportions of subunit 1Dx5 were much lower in the Anza lines studied here and by León et al. (2009) than in the lines studied in the previous reports. Comparison of line T618, expressing transgenes encoding subunits $1 \mathrm{Ax} 1,1 \mathrm{Dx} 5$ and $1 \mathrm{Dy} 10$ with a commercial variety (Perico) which contains the same endogenous $1 \mathrm{~A}$ and $1 \mathrm{D}$ subunits showed that the transgenic line had greater dough stability and strength. Although the two lines had similar proportions of glutenins, transgenic line T618 had a higher proportion of HMW-GS, with a correspondingly lower proportion of LMW-GS.

In conclusion, our results demonstrate that stacking HMW-GS transgenes by conventional crossing is a valid strategy for the improvement of wheat quality, with the differential effects being related to the different HMW-GS combinations. The presence of subunit 1Dy10 results in the formation of stronger dough regardless of the composition of $x$-type subunits. In patticular, the combination of subunits $1 \mathrm{Dx} 5$ and $1 \mathrm{Dy} 10$ results in greater dough strength than the other HMW-GS combinations.

\section{Acknowledgements}

The authors acknowledge funding by the Spanish C.l.C.Y.T. (project AGL2007-65685-C02-01). Rothamsted Research receives grant-aided support from the Biotechnology and Biological Sciences Research Council (BBSRC) of the UK. The technical assistance of Ana García and Azahara Vida is also acknowledged.

\section{References}

AACC, 2000. Physical Dough Tests. Mixograph Method, 10th ed. AACC. Method $54-40 A$.

Altpeter, F., Vasil, V., Srivastava, V., Vasil, I.K., 1996. Integration and expression of the higl molecular weight glutenin subunit $1 A \times 1$ gene into wheat. Nature Biotechnology 14, 1155-1159.
Alvarez, M.L., Gómez, M., Carrillo, J.M., Vallejos, R.H., 2001. Analysis of dough functionalicy of flours from transgenic wheat. Molecular Breeding 8, 103-108.

Barro, F., Barcelo, P., Lazzeri, P.A., Shewry, P.R., Ballesteros, J., Martin, A., 2003. Functional properties of flours from field grown transgenic wheat lines expressing the HMW subunit $1 \mathrm{Ax} 1$ and $1 \mathrm{D} \times 5$ genes. Molecular Breeding 12, 223-229.

Barro, F., Barcelo, P., Lazzeri, P.A., Shewry, P.R., Martín, A., Ballesteros, J., 2002. Field evaluation and agronomic performance of transgenic wheat. Theoretical and Applied Genetics $105,980-984$.

Barro, F., Rooke, L., Bekes, F., Gras, P., Tatham, A.S., Fido. R., lazzeri, P.A., Shewiy. P.R. Barcelo, P., 1997. Transformation of wheat with high molecular weight subunit genes results in improved functional properties. Nature Biotechnology 15. 1295-1299.

Blechl, A., Lin, J., Nguyen, S., Chan, R., Anderson, O.D., Dupont, F.M., 2007. Transgenic wheats with elevated levels of Dx5 andior Dy 10 high-molecular-weight glutenin subunits yield doughs with increased mixing strength and tolerance. Journal of Cereal science 45, 172-183.

Blechl, A., Anderson, O.D., 1996. Expression of a novel high-molecular-weight glutenin subunit gene in transgenic wheat. Nature Biotechnology 14, 875-879.

Butow, B.J., Tatham, A.S., Savage, A.W.J., Gilbert, S.M., Shewiy, P.R., Solomon, R.G. Bekes, F, 2003. Creating a balance - the incorporation of a HMW-GS subunit into transgenic lines. Journal of Cereal Science 38, 181-187.

Darlington, H., Fido, R., Tatham, A.S., Jones, H., Salmon, S.E., Shewry, P.R., 2003. Milling and baking properties of field grown wheat expressing HMW subunit transgenes. Journal of Cereal Science 38, 301-306.

Dick, J.A., Quick, J.S., 1983. A modified screening test for rapid estimation of gluten strength in early generation durum wheat breeding lines. Cereal Chemistry 60. 315-318.

Field, J.M., Bhandari, D., Bonet, A., Underwood, C., Darlington, H., Shewry, P., 2008 Introgression of transgenes into a commercial cultivar confilms diflerential effects of HMW subunits $1 \mathrm{Ax} 1$ and $1 \mathrm{D} \times 5$ on gluten properties. Journal of Cereal Science $48,457-463$.

Finney, K.F., Shogren, M.D., 1972. A ten-gram mixograph for determining and predicting functional properties of wheat flours. Baker's Digest 46, 32-47.

ICC. 1992a. Method for Using of the Chopin-Alveograph. International Association for Cereal Science and Technology. Method No. 121.

ICC. 1992b. Method for Using the Brabender Farinograph. International Association for Cereal Science and Teclinology. Method No. 115/1.

Larroque, 0.R., Gianibelli, M.C., Sanchez, M.G., MacRitchie, F., 2000. Procedure for obtaining stable protein extracts of cereal flour and whole meal for sizeexclusion HPLC analysis. Cereal Chemistry 77, 448-450.

León, E., Marín, 5., Giménez, M.J., Piston, F., Rodríguez-Quijano. M., Shewry. P.R. Barro, F, 2009. Mixing properties and dougl functionality of transgenic lines of a ommercial wheat cultivar expressing the 1Ax1, 1Dx5 and 1Dy10 HMW glutenin subunit genes. Journal of Cereal 5cience 49, 148-156.

Morel, M.H., Dehlon, P., Autran, J.C., Leygue, J.P., Bar-l'Helgouac'h, C., 2000. Elfects of temperature, sonication time and power settings on size distribution and extractabilicy of total wheat flour proteins as determined by size-exclusion high-performanc liquid chromatography. Cereal Chemistry 77, 685-691.

Payne, P.]., 1987. Genetics of wheat storage proteins and the efTect of allelic variation on bread-making quality. Annual Review of Plant Physiology 38, 141-153.

Rakszegi, M., Bekes, F., Lang, L, Tamas, L., Shewry, P.R., Bedo, Z., 2005. Technological quality of transgenic wheat expressing an increased amount of a HMW glutenim subunit. Journal of Cereal Science 42, 15-23.

Rooke, l., Bekes, F., Fido, R., Barro, F., Gras, P., Tatham, A.S., Barcelo, P., Lazzeri, P., Shewry, P.R., 1999. Overexpression of a gluten protein in transgenic wheat results in greatly increased dough strength. Joumal of Cereal Science 30 115-120.

Shewry, P.R., Halford, N.G., Lafiandra, D., 2003a. The genetics of wheat gluten proteins. Advances in Genetics 49, 111-184.

Shewry, P.R., Halford, N.G., Tatham, A.S., Popineau, Y., Lafiandra, D., Belton, P.S 2003b. The high molecular weight subunits of wheat glutenin and their role in determining wheat processing properties. In: Advances in Food and Nutrition Research. Academic Press, pp. 219-302.

Shewry, P.R., Tatham. A.5. Fido, R.J., 1995. Separation of plant proteins by electrophoresis. In: Jones, H. (Ed.), Methods in Molecular Biology: Plant Gene Transfer and Expression Protocols. Humana Press, Totowa, New Jersey, pp. 359-422.

Shuey. W.C. 1984. Interpretation of the Farinogram. In: D'Appolonia, B.L. Kunerth, W.H. (Eds.). The Farinograph Handbook. American Association of Cereal Chemistiy. 5t. Paul, MN, pp. 31-32. 